\title{
Surgical outcomes of diabetic hand infections in Lagos, Nigeria
}

\section{Bolaji O Mofikoya $^{1}$, Abdulwahab Ajani ${ }^{2}$, Andrew O Ugburo $^{1}$, Omotayo Olusoga ${ }^{3}$}

1. Burns, Plastic and Hand rehabilitation unit, Department of Surgery, College of Medicine,University of Lago Lagos Nigeria

2. Burns and Plastic Unit,Department of Surgery, Lagos State College of Medicine, Ikeja , Lagos , Nigeria

3. Burns, Plastic and Hand rehabilitation unit, Department of Surgery, Lagos University Teaching Hospital,Idiaraba, Nigeria
Date Received: 03-Jul-2018

Revision Received: 28-Sep-2018

Date Accepted: 10-Oct-2018

Correspondence: Bolaji Oyawale

Mofikoya

(bmofikoya@yahoo.com)

\section{Background:}

Abstract

Hand infections in diabetics can be a major cause of functional impairment. In patients with Tropical Diabetic hand syndrome prompt surgical intervention may salvage the hand, but return to premorbid function remain a challenge.

Objectives

A retrospective study of consecutive diabetic patients with hand infections was done in 2 tertiary institutions in Lagos, Nigeria to identify the epidemiology, modes of presentation, types of surgical intervention and outcomes of

treatment.

\section{Results}

Twenty one patients were studied over a 5 year period. ten males, eleven females. All were type 2 Diabetes. $52.4 \%(11)$ were diagnosed less than a year prior to presentation. 61.9\%(13) had digit/hand gangrene on presentation. All operated patients (20) had wound debridements. $52.5 \%$ (11) digit/hand amputations and this was the commonest surgical procedures done. Other surgeries done include skin grafts and 'flap reconstructions. There was a 19\% mortality rate. Less than a fifth were able return to their premorbid occupation after 3 months. But all had reduced range of motion in the interphalangeal joints in the adjacent digits.

\section{Conclusions}

There is a high morbidity rate for hand infections in the diabetic, even though majority of the hands were salvaged, most patients were unable to return to their premorbid occupation at 3 months.

Keywords: diabetes, infection, diagnosis, renal

\section{Introduction}

The World Health Organization (WHO) estimates that approximately 400 million people worldwide suffer from diabetes mellitus ${ }^{1}$. Over the last few decades, there has been an increasing incidence of severe hand infections in many diabetic patients ${ }^{2}$. These infections vary from localized septic lesions in a digit to fulminant limb gangrene with potentially fatal outcomes. Furthermore, it is now very common for many patients in the tropics to suffer the sequelae of severe hand impairments, including long-term morbidity.

While the entity of diabetic foot infection is well documented, little is known about similar infections in the hand ${ }^{3}$. However, it is believed that, as in the diabetic foot, the triad of poor vascularity, neuropathy and impaired cellular immunity may make the hand susceptible to infections in diabetic patients. Furthermore, this form of sepsis, occurring within tight compartments, renders the digits particularly susceptible to early-onset gangrene in some individuals. While foot infection has been estimated to be responsible for $9.7 \%$ of all hospital admissions, diabetic-related hand infections are said to make up $0.3 \%$ of all institutional admissions ${ }^{4}$. The term 'tropical diabetic hand syndrome' is often used to refer to patients who live in tropical or coastal areas with fulminant hand sepsis commonly resulting in the loss of hand function, amputation or even death (Figure 1 ). This syndrome is almost exclusively seen in the developing countries of Africa and some parts of Asia 5 . With Nigeria estimated to have the highest number of people with diabetes in Sub Saharan Africa, surgical outcomes of this important limb threatening complication would be of vital importance to clinicians in the region.

This study aims to determine the outcomes of surgical treatment in patients with diabetes-related hand infections in Lagos, Nigeria.

\section{Materials and methods}

We reviewed the medical charts of all patients above 18 years old with no prior upper extremity pathology or injury who were surgically treated for diabetic hand infections by the plastic surgery teams of two tertiary hospitals in Lagos, Nigeria between 2013 and 2017.This followed Institutional review board approvals in both institutions. We also reviewed data relating to the clinical evaluation of hand function for 3 months post-surgery. Patients were excluded if they had incomplete medical records or failed to undergo functional evaluation over for 3 months post-surgery . All patients had received post-operative hand therapy, which commenced as soon as possible after wound healing. This therapy consisted of supervised hand exercises, wax therapy, ultrasonic scar massage and other individualized treatments, as needed. This therapeutic approach aimed to restore the full range of motion in the hand. 
Medical charts were reviewed for all patient demographic data, the type of diabetes, the duration of diagnosis, hand involvement, comorbidities, the type and extent of infection and X-ray findings. Microscopy, culture and sensitivity tests were carried out on swabs taken from all wounds. We also recorded the types of surgical intervention, complications arising after surgery and mortality. We also assessed the extent of hand use 3 months after surgery as well as the ability to return to pre-morbid occupational activities. All data were entered using SPSS version 21.0. Released 2012. IBM Statistics for windows.Armonk NY: IBM Corp. and confidence levels set at $95 \%$.

\section{Results}

Thirty-five patients with diabetes-related hand infections were treated at the two centres over the 5-year study period. This compared with 844 patients with diabetic foot lesions over the same period of time. None of the patients had concomitant hand and foot infections, although one patient had sequential infections that necessitated amputations in two adjacent fingers on the same hand; these infections occurred 18 months apart. Only 21 of the 35 patients had a complete set of data and underwent full functional evaluation during the follow-up period. Of these 21 patients, 10 were male and 11 were female. Mean patient age was $47.4 \pm 2.8$ years (mean \pm standard deviation). All of the 21 patients had type 2 diabetes mellitus; the left hand was involved in 9 patients and the right hand in 11 patients. Eleven of the patients presented with hand infection less than a year after the diagnosis of diabetes. The mean duration of diabetes prior to the presentation of hand infection was $4.7 \pm 1.3$ years. Only one patient was not previously diagnosed with diabetes prior to the hand infection. Four of the 11 patients had associated hypertension and 6 had clinical and biochemical evidence of renal impairment; 2 of the patients with renal impairment were undergoing dialysis. Of the 6 patients with renal impairment, 3 died during the course of treatment. Mean blood glucose on presentation was $481 \pm 78 \mathrm{mg} / \mathrm{dl}$ ); 13 patients had deep ulcers with localized digital gangrene (Table 1).

Microbial cultures grown from the swabs of 10 patients grew a range of microorganisms in 10 patients; Staphylococcus and Klebsiella species were the most commonly isolated organisms. Other organisms are shown in Table 2. Swabs taken from the remaining 11 patients did not grow any organisms on aerobic culture. Anaerobic and fungal cultures were not carried out. Over half $(57.1 \%)$ of the patients seen presented with digital gangrene while most of the remaining patients had extensive ulcers with deep subcutaneous abscess collections (Table 1). All of the patients included in this study required two or more rounds of surgery and needed initial debridement; the majority of the patients required a ray amputation (Table 3). Two patients needed reverse radial forearm flaps; in both cases, the two flaps suffered from partial necrosis but did not require further intervention. One patient required multiple ascending amputations to control fulminant hand sepsis, although this wound eventually healed following a below-elbow amputation.

Four patients died during the study. The causes of death were end-stage renal impairment (3 patients) and overwhelming septicaemia (in 1 patient). The evaluation of early functional outcome at 3 months showed that only 4 patients had returned to their premorbid occupation. There was universal residual stiffness in all patients, particularly at the metacarpophalangeal joint. All patients with single digit amputations that did not involve the thumb believed that they had coped well with activities of daily living but admitted to significant interphalangeal joint stiffness in the adjacent digits.

Table 1. Wagner ${ }^{20}$ classification of hand infection in a study population of 21 diabetic patients

\begin{tabular}{|l|l|l|}
\hline Class & Lesion & Frequency (\%) \\
\hline 1 & Superficial ulcer & $1(4.7)$ \\
\hline 2 & $\begin{array}{l}\text { Ulcer with ligament tendon capsule } \\
\text { involvement }\end{array}$ & $6(28.6)$ \\
\hline 3 & Bone involvement & - \\
\hline 4 & Localized gangrene & $13(61.9)$ \\
\hline 5 & Extensive hand gangrene & $1(4.7)$ \\
\hline
\end{tabular}

Table 2. Results arising from microbial cultures

\begin{tabular}{|l|l|}
\hline Organism & Number of patients $^{\mathbf{a}}$ \\
\hline Staphylococcus aureus & 7 \\
\hline Klebsiella spp. & 5 \\
\hline Proteus mirabilis & 5 \\
\hline Escherichia coli & 2 \\
\hline Acinetobacter spp. & 1 \\
\hline
\end{tabular}

Table 3. Surgical interventions in the study population

\begin{tabular}{|l|l|}
\hline Type of surgery & Number of patients (\%) \\
\hline Debridement & $20(95.3)$ \\
\hline Ray amputation of the digit & $11(52.3)$ \\
\hline $\begin{array}{l}\text { Drainage of deep subcutaneous } \\
\text { abscesses }\end{array}$ & $4(19)$ \\
\hline Skin graft & $2(9.5)$ \\
\hline Flap reconstruction & $2(9.5)$ \\
\hline Hand amputation & $1(4.7)$ \\
\hline
\end{tabular}

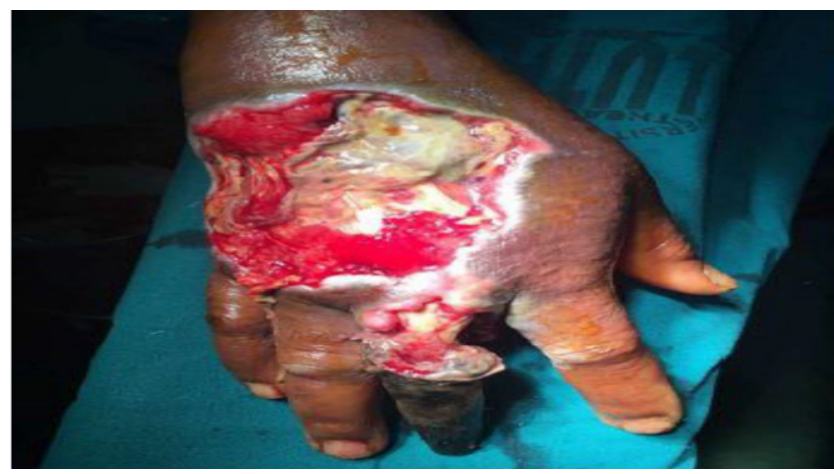

Figure 1: Fulminant diabetic infection of the right hand with gangrene of the middle finger

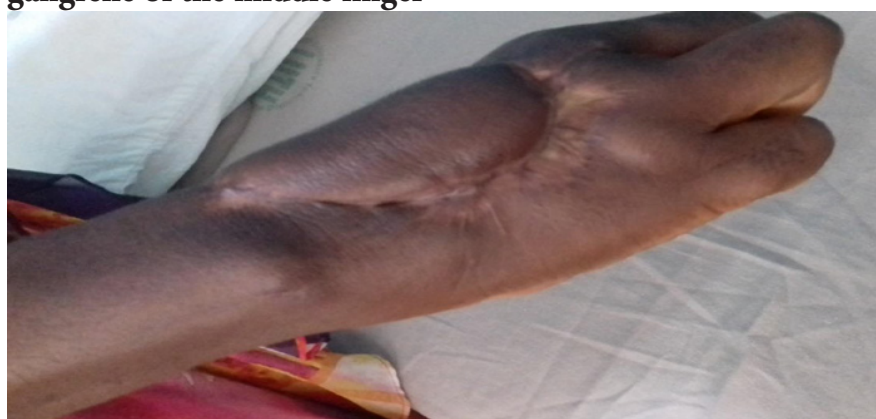

Figure 2: Same patient following debridement, ray amputation of the middle finger and flap cover for the dorsal hand defect. Note metacarpophalangeal joint stiffness 


\section{Discussion}

The occurrence of hand infections in patients with diabetes mellitus has been well documented in literature from the western world ${ }^{6}$. However, the occurrence of aggressively destructive hand infections in tropical countries is a relatively recent finding 7,8 . We sought determine the outcomes of this condition following surgical intervention.

Our present study population was dominated by females; this is interesting as this concurs with previous literature from African countries ${ }^{9,10}$. Conversely, several previous reports from Asian countries have reported male dominance ${ }^{11}$. These observations are believed to be due to the fact that in many African communities, women make up the majority of the manual labour work force as opposed to men in Southwest China and India ${ }^{11,12}$. Consequently, African females are more likely to suffer minor hand trauma that could predispose these individuals to infections.

The mean duration of diabetes prior to the presentation of hand infection was $4.7 \pm 1.7$ years in our patients. This was shorter than that described in most other reports; for example, 5.3 years in Southwest China, 7.0 years in South India and 8.2 years in Turkey ${ }^{11-13}$. This finding might be related to poor compliance with medications during the early stages of the disease following diagnosis. This is further reinforced by the fact that 11 of our 21 patients presented within 1 year of diagnosis.

While many researchers have reported amputation rates ranging from $13.9 \%$ in Northern Nigeria to $16 \%$ in Sudan and Hong Kong ${ }^{14-16}$, two-thirds of our present cohort of patients presented with gangrene (digits in 13 patients and the hand in 1 patient), thus necessitating digit or forearm amputations. This could be explained by late presentation as over half of our patients presented with overt gangrene. The late presentation is most probably due to low levels of education regarding diabetic infections and poor awareness of the potential severity of diabetic hand infections among primary healthcare providers. Limited access to healthcare facilities, on account of cost issues, creates further delays in patients receiving prompt surgical intervention. Moreover, several of our patients may have delayed consent to surgery due to fear of limb loss.

In 2 of our patients, complex dorsal skin loss necessitated a reverse radial forearm flap cover; unfortunately, both flaps suffered a partial loss. This observation was similar those made by previous researchers, who reported that up to a third of their patients also had some flap loss. This is not surprising as both macro- and micro-angiopathy have been well documented in diabetic limbs and this can affect the survival of reverse flow flaps. Another study reported a 20\% partial necrosis rate following the use of this form of flap in diabetic patients ${ }^{17}$; in this previous study, re-grafting was not required, as was the case in our own study.

In the present study, we found that renal impairment and hypertension were the main accompanying comorbidities (47.2\%); this finding concurred with previous research ${ }^{13}$. These factors may have been responsible for the high mortality rate $(19 \%)$ in our patient cohort. Three patients with associated end stage renal disease died during treatment due to complications arising from renal failure. One other patient died of septicaemia. It has been well documented that renal impairment is associated with higher rates of amputation and mortality in diabetic patients ${ }^{17,18}$.
Recent reports describe a reduction in the morbidity and mortality associated with diabetic hand infections. The factors underlying this reduction are earlier diagnosis, better glycaemic control and more aggressive surgical treatment in the Western world ${ }^{19}$. In selected centres, early presentation, the use of vacuum-assisted wound closure, and hyperbaric oxygen therapy, also appear to lead to improvements in functional outcome, as well as hand salvage rates ${ }^{20}$. Upon evaluating hand function at 3 months post-surgery, we found universal ankylosis of the metacarpophalangeal(MCPJ) and proximal interphalangeal(PIPJ) joints in the affected hands (Figure 2). Mean range of motion was 20 degrees with a standard deviation of 8 degrees at the metacarpophalangeal joint(MCPJ).

Very little previous research has attempted to assess hand function following hand infection in diabetic patients, although we do know that the extent of residual stiffness, even after the completion of hand therapy, can be as high as $21 \%{ }^{15}$. At the time of evaluation, the majority of our patients were still undergoing hand therapy. Furthermore, only $19 \%$ of our patients had returned to their premorbid occupation by the time of evaluation. All patients with minor amputations believed they had been coping with most activities of daily living but admitted to limitations with regards to tasks requiring precise finger motion and pickingup small objects in the affected hand. Other researchers have reported functional impairment in up to $72.4 \%$ of patients, although the precise nature of such impairment was not specified $^{21}$.

Our study had limitations which need to be considered. For example, this study was based on a tertiary centre; this may be responsible for the rates of morbidity and mortality among our patient cohort. Other limitations include the small sample size and the short follow-up period.

In conclusion, diabetic hand infections in the Lagos area, tend to present with early onset tissue gangrene in middleaged patients often with significant perioperative mortality. Our work showed considerable functional limitations of salvaged limbs. It is hoped that a future study involving a larger cohort will further highlight the independent predictors of such high morbidity in our patient population.

\section{Authors' contributions}

Study conceptualized by BOM and AA, data collected by all authors. All authors read and approved of final manuscript.

\section{Funding- nil}

Data and materials- available for review

\section{Competing interests-none References}

1. WHO Global Report on Diabetes. 2016. WHO Cataloguing-in publication data. ISBN 9789241565257 (NLM classification: WK 810).

2. Betotmane A, Faraoun K, Mohhamedi F, Benkhelifa T, Amani ME. Infections of the upper extremity in hospitalized diabetic patients, a prospective study. Diabetes Metab. 2004;30:91:7.

3. Gill GV, Famuyiwa OO, Rolfe M, Archibald LK. Tropical diabetic hand syndrome. Lancet. 1998;35;9096:113-4.

4. Abbas ZG, Lutale J, Archibald LK, Jarvis WR, Beckles G, Moore K. Tropical diabetic hand syndrome - Dar es Salaam, Tanzania, 19982002. MMWR Morb Mortal Wkly Rep. 2002;51:969-70.

5. Stevenson J, Anderson IVR. Hand infections: An audit of 160 
infections treated in an accident and emergency department. J Hand Surg Br. 1993;18:115-8.

6. Mann RJ, Peacock JM. Hand infections in patients with diabetes mellitus. Trauma. 1977;17:376-80.

7. Akhuemokhan K, Echekwube P, Bakpa F, Okokhere P. Tropical diabetic hand syndrome: prevention through education. S Afr J Clin Nutr. 2011;24:205-6.

8. Gill GV, Famuyiwa OO, Rolfe M, Archibald LK. Serious hand sepsis and diabetes mellitus: specific tropical syndrome with western counterparts. Diabet Med. 1998;15:858-62.

9. Akintewe TA. The diabetic hand-5 illustrative case reports. Br J Clin Pract 1984;38:368-71.

10. Bosseri S, Gill G. Hand and foot sepsis in Libyan diabetic patients. Trop Doct 1997;27:232-233.

11. Wang C, Lv L, Wen X, Chen D, Cent S, Huang H, et al. A clinical analysis of ulcer in a diabetic foot center. Diabetes Med. 2010;27:84851. DOI: 10.1111/j.1464-5491.2010.03012.x.

12. Raveendran S, Naik D Raj Pallapatti SC, Prakash JJ, Thomas PB, Thomas S. The clinical and microbiological profile of the diabetic hand: a retrospective study from South India. Indian J Endocrinol Metab. 2016;20:5:619-24. DOI: 10.4103/2230-8210.190539.

13. Ince B, Dadaci M, Arslan A, Altuntas Z, Evrenos MK, Karslis MF. Factors determining poor prognostic outcomes following diabetic hand infections. Pak J Med Sci. 2015;31:532-37. DOI: 10.12669/ pjms.313.6858.
14. Lawal YZ, Ogirima MO, Dahiru IL, Girei BA, Salisu MB. Tropical diabetic hand syndrome: Surgical management and proposed classification. Arch Int Surg 2013;3:124-7.

15. Hamed ME, Mahmoud SM, Mahadi S, Widatalla AH, Shawir MA, Ahmed

ME. Hand sepsis in patients with diabetes mellitus. Saudi Med J. 2009;30:1454-8.

16. Jalil A, Barlaan PI, Fung BKK, Ip JWK. Hand infection in diabetic patients. Hand Surg. 2011;16:307-12. DOI: 10.1142/ S021881041100559X.

17. Helmy Y, Taha, A, El-Banooby T, Khallaf NA, Moawad M. Reconstruction of dorsal diabetic hand defects by reverse radial forearm flap. Int J Curr Res. 2017;9:45739-42.

18. Francel TJ, Marshall KA, Savage RC. Hand infections in diabetics and diabetic renal transplant patients. Ann Plast Surg. 1990;24:304-9.

19. Pinzur MS, Bednar M, Beaver F, Williams A. Hand infections in the diabetic patient. J Hand Surg Br. 1997;22: 133-4.

20. Aydin F, Kaya A, Savran A, Incesu M, Karakuzu C, Ozturk AN. Diabetic hand infections and hyperbaric therapy. Acta Orthop Traumatol Turc. 2014;48:649-54.

21. Sidibe AT, Dembele M, Cisse A, Alwata F, Ould Ahmedou MY, Coulibaly $\mathrm{T}$, et al. Diabetic hand infections in hospital practice in Bamako Mali. Diabetes Metab. 2006;32:89. 